> L'un des mécanismes de défense de l'hôte consiste en l'immunité entraînée, composante de l'immunité innée, également connue sous le nom de mémoire immunitaire innée. La mémoire immunitaire innée est définie comme une protection exacerbée d'un organisme lors d'un deuxième contact avec un corps étranger, comme un microorganisme pathogène. II s'agit d'une immunité qui ne fait pas intervenir les composants de l'immunité acquise, comme les lymphocytes B ou les lymphocytes $\mathrm{T}$. Elle repose essentiellement sur la machinerie cellulaire et moléculaire de la réponse innée. La mémoire immunitaire innée a été décrite aussi bien chez les invertébrés et que les vertébrés, mais son mécanisme ne commence à être élucidé que depuis récemment, en particulier chez les vertébrés. La plupart des recherches sur la mémoire immunitaire innée repose, aujourd'hui, sur des modèles vertébrés, oubliant que ce mécanisme existe également chez les invertébrés. Pourtant, les invertébrés présentent l'avantage de pouvoir étudier la mémoire immunitaire dans des modèles qui sont naturellement dépourvus d'immunité acquise. L'objectif de cette revue est donc de réaliser un point sur la mémoire immunitaire innée des invertébrés. <

L'immunité est scindée généralement selon deux catégories de réponse : l'immunité adaptative et l'immunité innée, chacune ayant des propriétés propres, même si elles interagissent. L'immunité adaptative, considérée comme complexe, présente une spécificité et une mémoire. L'immunité innée, quant à elle, est décrite pour être plus simple, du fait de sa non spécificité et de l'absence de mémorisation. Les travaux menés ces dernières années sur l'immunité innée ont cependant montré la fragilité de cette dichotomie. L'immunité innée se révèle être, en effet, plus complexe puisqu'il

Vignette (Photo @ Inserm-Fathia Mami-Chouaib).
La mémoire immunitaire entraînée chez les invertébrés

\section{Que sait-on?}

Cédric Torre, Landry Laure Tsoumtsa, éric Ghigo

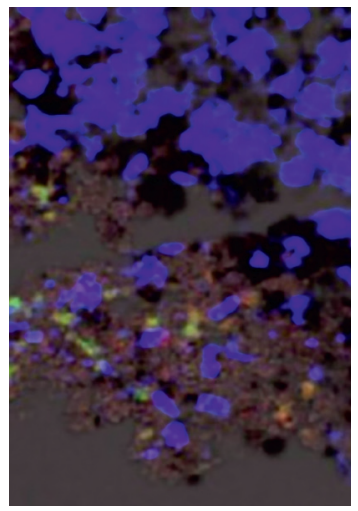

URMITE (Unité de recherche sur les maladies infectieuses et tropicales émergentes), CNRS UMR 7278, IRD 198, Inserm U1095, APHM, Institut hospitalier universitaire MéditerranéeInfection, Aix-Marseille Université, 19-21, boulevard Jean Moulin, 13385 Marseille Cedex 05, France.

eric.ghigo@univ-amu.fr

est fortement suggéré qu'elle serait douée d'une mémoire potentiellement spécifique : cette caractéristique a été nommée trained immunity (immunité entraînée) par Mihai Netea et ses collègues de l'université de Radboud (Nimègue, Pays-Bas), en 2011 [1]. Elle est également appelée «mémoire immunitaire innée ». L'existence de cette mémoire immunitaire innée a été décrite chez les plantes [2, 3], les invertébrés [4-7] et les vertébrés [1]. Elle se caractérise par une réponse de l'hôte qui est potentialisée lors d'un second contact avec un agent pathogène, ou ses constituants (Figure 1). Le mécanisme de mémoire immunitaire innée, bien que similaire dans son principe à la mémoire immunitaire de type adaptatif, reste différent de par les processus moléculaires mis en jeu. $\varepsilon n$ effet, chez les mammifères, au contraire de la mémoire adaptative, la mémoire immunitaire innée ne fait pas appel à des modifications génétiques permanentes comme la recombinaison $V(D) J$ des gènes codant les domaines variables d'immunoglobulines ou le TCR ( $T$ cell receptor), mais elle nécessite une reprogrammation des fonctions cellulaires par des mécanismes épigénétiques $[1,8]$.

La mémoire immunitaire innée chez les vertébrés a fait l'objet de nombreuses revues, ce qui n'est pas le cas pour les invertébrés. Nous aborderons dans cette revue la mémoire immunitaire innée chez les mammifères, afin de poser brièvement les bases biologiques du processus. Nous nous focaliserons ensuite sur la mémoire immunitaire innée chez les invertébrés.

\section{Mémoire immunitaire innée chez les vertébrés}

Dans les années 1980, F. Bistoni et ses collègues, de l'université de Perugia, en Italie, avaient observé que des souris inoculées avec 


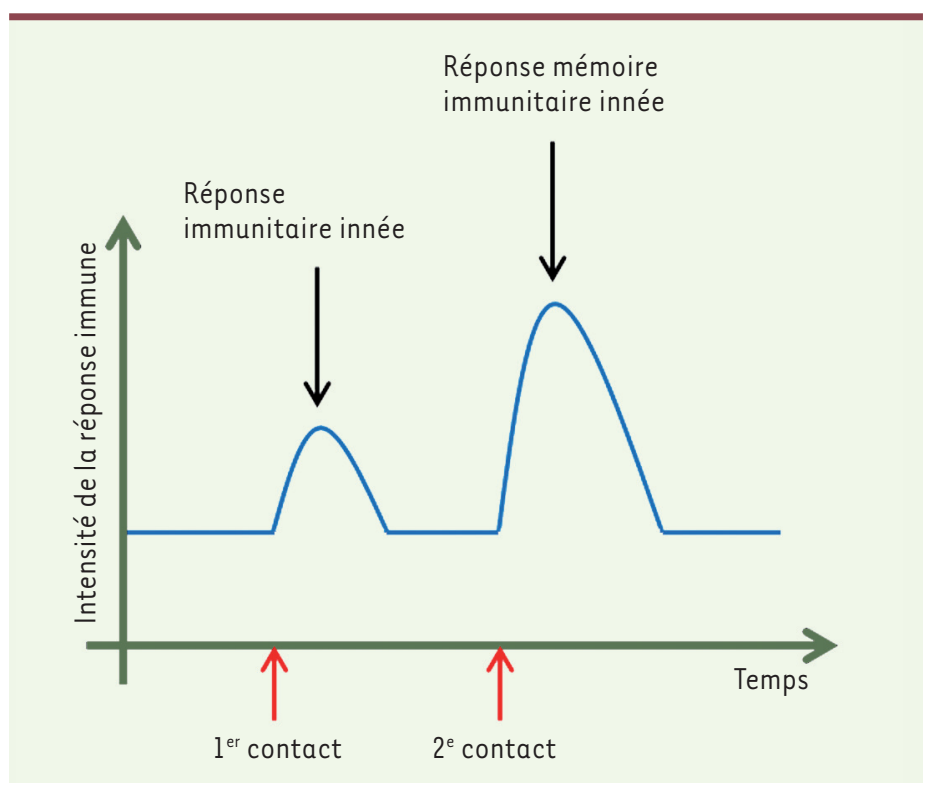

Figure 1. La mémoire immunitaire innée. Illustration représentant l'augmentation de l'intensité de la réponse immune à la suite d'un premier, puis un second, contact avec un microbe induisant une réponse immune. La mémoire immunitaire innée correspond à une réponse potentialisée, réduisant la susceptibilité de l'hôte à l'infection.

une souche atténuée de levure Candida albicans développaient une protection contre la levure, mais également contre des bactéries comme Staphylococcus aureus [9]. Cette protection ne nécessitait pas la présence de lymphocytes T [10], mais elles dépendait des macrophages [9] et de la production de cytokines de type pro-inflammatoire [11]. Différentes études ont, depuis, permis de définir les mécanismes régissant la mémoire immunitaire innée chez les vertébrés. L'utilisation de souris génétiquement modifiées [12], afin qu'elles soient dépourvues de système immunitaire adaptatif, a ainsi permis d'établir que la mémoire immunitaire innée ne requérait pas les lymphocytes $T$ et $B$, mais nécessitait des cellules effectrices de l'immunité innée comme les monocytes/macrophages [12,13] et les cellules NK (natural killer) [14]. L'établissement d'une mémoire immunitaire innée repose sur l'engagement, par les pathogen-associated molecular patterns (PAMP), des molécules présentes à la surface des agents microbiens, de récepteurs particuliers exprimés par ces cellules, les pattern recognition receptors (PRR), comme les Toll-like receptors (TLR), les lectines de type C, les NOD (nucleotide oligomerization domain)-like receptors (NLR), et les RIG (retinoic acid-inducible gene)-like receptors (RLR), [13, 15-17]. Le mécanisme fait intervenir une reprogrammation épigénétique de la cellule par la modification, par méthylation et acétylation, des histones autour desquelles se compacte l'ADN (Figure 2) [8]. Les marques épigénétiques, ainsi produites, qui sont les plus fréquentes, sont l'H3K4mel (c'est-à-dire la mono-méthylation de la lysine en position 4 de l'histone $\mathrm{H3}$ ), l'H3K4me3 (la tri-méthylation de cette même lysine) et l'H3K27ac (I'acétylation de la lysine 27 de l'histone IH3)
[18]. Cette reprogrammation épigénétique modifie la charge des histones. Elle conduit à une forme ouverte de la chromatine, propice au fonctionnement de la machinerie transcriptionnelle et à l'expression des gènes. Les modifications des histones peuvent également stabiliser la liaison de protéines à la chromatine sans nécessairement altérer sa structure [19]. Des acteurs de la réponse immunitaire comme le TNF- $\alpha$ (tumor necrosis factor) ou l'IFN- $\gamma$ (interféron gamma), pourraient ainsi voir leur production potentialisée. Une base métabolique a également été suggérée dans le processus à l'origine de la mémoire immunitaire innée [18]. En effet, si le métabolisme cellulaire du glucose est important pour la réponse immunitaire aiguë [20], plusieurs métabolites du métabolisme glucidique apparaissent comme des co-facteurs d'enzymes qui sont impliquées dans les modifications épigénétiques [21]. Un lien étroit entre glycolyse, phosphorylation oxydative et tolérance immunitaire a également été décrit [22, 23].

\section{Mémoire immunitaire innée chez les invertébrés}

Les organismes primitifs que sont les invertébrés possèdent un système immunitaire inné mais ils sont dépourvus de système immunitaire adaptatif [24]. Ils constituent donc un modèle de choix pour l'étude de la réponse immunitaire innée et, en particulier, de la mémoire immunitaire innée (Tableau 1). En effet, l'ensemble des acteurs de la réponse immunitaire adaptative ne sont naturellement pas présents chez les invertébrés. Ils ne peuvent donc pas influer sur les mécanismes mis en jeu dans la mémoire immunitaire innée, permettant ainsi une approche simplifiée des processus et de leur identification.

Différentes expériences d'allogreffes, qui ont été conduites sur les éponges, représentantes de l'embranchement des Porifera situé au plus bas des métazoaires, ont révélé l'existence d'une mémoire immunitaire innée [25]. Ainsi, en 1979, la présence d'une mémoire immunitaire innée chez Callyspongia diffusa a été mise en évidence grâce au rejet d'allogreffe : alors que l'isogreffe n'induit pas de rejet chez C. diffusa, la greffe entre éponges de la même espèce est incompatible. Le temps de rejet observé après une première, une seconde et une troisième allogreffe, diminue, ce qui suggère l'existence d'une mémoire induite par la succession des greffes [25]. De tels résultats ont également été obtenus avec le corail, Montipora verrucosa [26], et le ver de terre, Lumbricus terrestris [27]. Ces organismes invertébrés développent donc une mémoire immunitaire innée induite par un premier stimulus représenté ici par la greffe. 


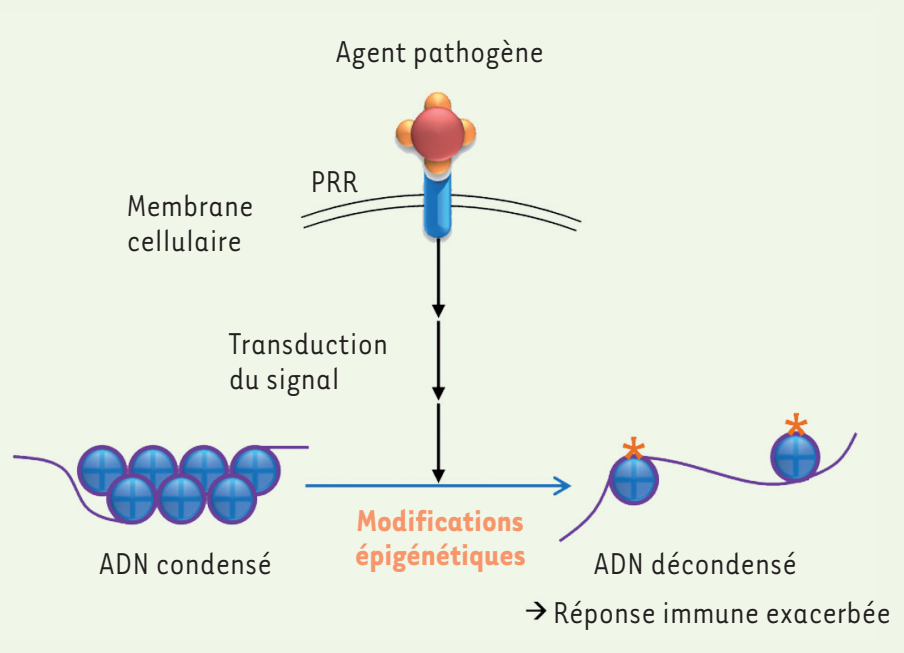

Figure 2. Mécanisme intracellulaire de l'établissement de la mémoire immunitaire innée. L'activation d'un PRR (pattern recognition receptor) à la surface d'une cellule de l'immunité innée par un agent pathogène induit une cascade de transduction du signal qui aboutit à des modifications épigénétiques permettant, lors d'un second contact, une réponse immune exacerbée.

Chez les invertébrés, la mémoire immunitaire innée peut également être initiée à la suite d'interactions avec un pathogène. Elle est alors induite lors d'un premier contact avec le microorganisme, et peut présenter une certaine spécificité $[1,28]$. La littérature fourmille d'exemples d'induction d'une mémoire innée plus ou moins spécifique chez les invertébrés. Cependant très peu ont été étudiés en détail. L'insecte Periplaneta americana (ou blatte américaine) développe une mémoire en réponse à une infection par la bactérie Pseudomonas aeruginosa. Cette mémoire ne présente toutefois une spécificité de reconnaissance que lorsque l'infection est réalisée avec un extrait de la bactérie. En effet, la bactérie entière n'induit pas de réponse mémoire. En revanche, l'insecte injecté avec d'autres bactéries (Serratia marcescens, Streptococcus lactis ou Micrococcus lysodeikticus) est protégé contre une infection par P. aeruginosa [29]. Drosophila melanogaster (la mouche du vinaigre) est protégée par une primoinfection induite avec des doses sub-létales de Streptococcus pneumoniae, d'une infection secondaire réalisée avec une dose létale de la même bactérie [7]. Toutes les bactéries ne sont pas à l'origine de l'induction chez l'hôte d'une protection contre une seconde infection. En effet, une primo-infection par Salmonella typhimurium, Listeria monocytogenes et Mycobacterium marinum, inactivées par la chaleur, ne protège pas la drosophile contre une seconde infection par ces mêmes bactéries virulentes. L'insecte, ayant développé une mémoire contre S. pneumoniae, n'est également pas protégé contre une infection par d'autres microbes. Il existe donc un certain niveau de spécificité de reconnaissance pour la mémoire innée développée au cours des infections bactériennes.

Chez la drosophile, la présence de phagocytes, ainsi qu'un processus de phagocytose efficace, sont nécessaires à l'établissement d'une mémoire immunitaire innée. Une délétion de la voie de signalisation
Imd ${ }^{1}$ n'affecte pas l'installation de la mémoire lors de la primo-infection par S. pneumoniae. Par contre, les drosophiles déficientes pour des gènes codant des protéines impliquées dans la voie de signalisation Toll, comme DIF (dorsal-related immunity factor), I'homologue de NF- $\kappa \mathrm{B}$, ou PGRP-SA (peptidoglycan recognition protein SA) [39] $(\rightarrow)$, un récepteur du peptidoglycane bactérien, ne sont

$(\rightarrow)$ Voir la Dernière heure de J. Royet, $\mathrm{m} / \mathrm{s}$ $n^{\circ} 12$, décembre 2001, page 1359

plus capables de développer une mémoire immunitaire innée [7]. Macrocyclops albidus, un crustacé plus communément appelé cyclope à cornes grêles, développe une mémoire immunitaire innée lorsqu'il a été exposé à son parasite naturel, le ver Schistocephalus solidus [4], qui le protège contre l'infection par des parasites de la même espèce. Ce mécanisme pourrait reposer sur la reconnaissance du parasite par des lectines exprimées par le cyclope, mais cela n'a jamais été étudié [4]. Chez Porcellio scaber, un autre crustacé, un premier contact avec la bactérie Bacillus thuringiensis, ou avec Escherichia coli tuée par la chaleur, stimule la phagocytose par les hémocytes (équivalents des phagocytes) vis-à-vis de la même bactérie, lors d'un second contact [30]. Cette mémoire présente une certaine spécificité. Le système immunitaire de $P$. scaber est en effet capable de différencier les bactéries Gram négatif des bactéries Gram positif. Il peut également distinguer des bactéries pathogènes et des bactéries qui ne le sont pas, et différentes espèces de bactéries à Gram positif. Les mécanismes déterminant cette spécificité restent à élucider. Néanmoins, il semble que les hémocytes soient à l'origine de ces distinctions, de même que la régulation différentielle de l'expression de peptides antimicrobiens, via les voies de signalisation impliquant Imd et Toll [31]. Chez la planaire Dugesia dorotocephala (ou Plathelminthe) $[40](\rightarrow)$, une première infection

$(\rightarrow)$ Voir la Nouvelle de C. Torre et $\varepsilon$. Ghigo, $m / s n^{\circ} 1$, par des mycobactéries (Mycobacjanvier 2015, page 20 tuberculosis) inactivées par la chaleur, conduit, par la mobilisation des cellules réticulaires ${ }^{2}$ après une deuxième infection, à une élimination plus rapide des bactéries mortes [32]. Bien que cette observation n'ait pas été définie comme relevant d'une mémoire immunitaire innée, elle en présente certaines caractéristiques qui font de la planaire un organisme également doué de

${ }^{1}$ IMD est une protéine à « death domain » similaire à la RIPl (receptor-interacting protein 1) de la voie du TNF- $\alpha$ des mammifères. IMD se lie à l'homologue de la molécule FADD des mammifères ( $\mathrm{dFADD}$ ) qui interagit ensuite avec DREDD (homologue de la caspase 8).

${ }^{2}$ Chez la planaire, il n'est pas prouvé que ce soit des hémocytes. Peut-être s'agit-il de deux types cellulaires distincts. 


\begin{tabular}{|c|c|c|c|c|}
\hline Embranchement & Espèce & Intervention/Infection & Spécificité & Références \\
\hline Porifera & Callyspongia diffusa & Transplantation tissulaire & Oui & [25] \\
\hline Cnidaria & Montipora verrucosa & Transplantation tissulaire & Oui & {$[26]$} \\
\hline Annelida & Lumbricus terrestris & Transplantation tissulaire & Oui & [27] \\
\hline Crustacea & Daphnia magna & Pasteuria ramosa & Oui & {$[5]$} \\
\hline Crustacea & Macrocyclops albidus & Schistocephalus solidus & Oui & [4] \\
\hline Crustacea & Porcellio scaber & Bacillus thuringiensis, Escherichia coli & Oui & {$[30]$} \\
\hline Insecta & Periplaneta americana & Pseudomonas aeruginosa & Oui & [29] \\
\hline Insecta & Drosophila melanogaster & Streptococcus pneumoniae & Oui & [7] \\
\hline Insecta & Bombus terrestris & $\begin{array}{l}\text { Pseudomonas fluorescens, Paenibacil- } \\
\text { lus alvei, Paenibacillus larvae }\end{array}$ & Oui & [6] \\
\hline Plathelminthe & Dugesia dorotocephala & $\begin{array}{l}\text { Mycobacterium tuberculosis, Mycobac- } \\
\text { terium bovis }\end{array}$ & ND & [32] \\
\hline
\end{tabular}

Tableau I. Exemples d'organismes modèles invertébrés ayant une mémoire immunitaire innée. ND : non déterminé.

mémoire immunitaire innée. Là encore, le mécanisme reste inconnu, mais la phagocytose semble jouer un rôle important.

La possibilité d'un transfert intergénérationnel de la mémoire immunitaire innée a été envisagé. En effet, le cyclope $M$. albidus ayant développé une mémoire immunitaire innée contre $S$. solidus transmet cette protection à sa descendance [4]. Il en est de même pour la puce d'eau, Daphnia magna (un crustacé), pour l'infection par Pasteuria ramosa [5]. Le bourdon terrestre (Bombus terrestris), qui développe une mémoire immunitaire innée spécifique à l'infection par Pseudomonas fluorescens, est aussi capable de transférer son immunité à sa descendance $[6,33,34]$, celle-ci faisant intervenir la phénoloxydase de l'hémolymphe.

\section{Conclusion et perspectives}

Les invertébrés sont capables de développer une mémoire immunitaire innée qui les protège spécifiquement contre des microorganismes pathogènes rencontrés lors d'un premier contact. Les mécanismes de cette mémoire restent peu compris. Le programme épigénétique et son effet sur la transcription des gènes possiblement impliqués n'ont que peu été étudiés. Le processus ferait intervenir la phagocytose. Elle jouerait un rôle important dans l'établissement de la mémoire immunitaire innée avec l'implication des phagocytes. Les recherches actuelles se sont focalisées sur l'implication de ces cellules, par analogie avec celles des vertébrés. D'autres types cellulaires, beaucoup moins professionnels en termes de capacité immunitaire, pourraient néanmoins être impliqués. Le transfert intergénérationnel de la mémoire immunitaire innée suggère l'implication de cellules qui pourraient être des cellules somatiques mais aussi germinales. Le rôle de ces différentes cellules pourrait être examiné en utilisant certains invertébrés et leurs capacités biologiques, comme la planaire, qui présente un taux de cellules souches important (entre 20 et $30 \%$ ) [35], ou le tardigrade ${ }^{3}$, capable de survivre à des environnements hostiles [36-38]. La compréhension des mécanismes impliqués dans la mise en place d'une mémoire innée chez ces organismes, conservés au cours de l'évolution, devrait permettre de mieux les appréhender dans les organismes plus complexes que sont les vertébrés et, ainsi, d'envisager de nouvelles stratégies de lutte contre les microbes pathogènes. $\diamond$

\section{SUMMARY}

Trained immunity in invertebrates: what do we know? One of the defense mechanisms of the host is the trained immunity, an immune component of the innate immunity, also known as innate immune memory. The trained immunity is defined as an exacerbated protection of an organism to a foreign body, such as a pathogenic microorganism, upon a second contact with it. This kind of immunity does not involve the components of acquired immunity, such as the B lymphocytes or T lymphocytes. It relies heavily on the cellular and molecular machinery of the innate response. Trained immunity is described as being present in both invertebrates and vertebrates, but its mechanisms have only recently begun to be elucidated, particularly in the vertebrates. Today, most of the research about innate immune memory deals with vertebrate models, forgetting that

\footnotetext{
${ }^{3}$ Proche des Arthropodes. II mesure un peu plus de $1 \mathrm{~mm}$ en moyenne et il est le seul animal connu qui peut survivre dans l'environnement hostile du vide spatial. II peut également résister à des températures proches du zéro absolu et jusqu'à $150^{\circ} \mathrm{C}$.
} 
this mechanism exists in invertebrates. Investigating trained immunity in invertebrates gives the advantage to work with models naturally lacking acquired immunity. The aim of this review is to take stock of the knowledge concerning the trained immunity entailed in invertebrates. $\diamond$

\section{REMERCIEMENTS}

Cédric Torre est financé par le Ministère de l'Enseignement Supérieur et de la Recherche (MESR, 2013-2016) et par la Fondation pour la Recherche Médicale (FRM, FDT20160435255). Landry Laure Tsoumtsa est financée par la Fondation Méditerranée Infection.

\section{LIENS D'INTÉRêT}

Les auteurs déclarent n'avoir aucun lien d'intérêt concernant les données publiées dans cet article.

\section{RÉFÉRENCES}

1. Netea MG, Quintin J, van der Meer JW. Trained immunity: a memory for innate host defense. Cell Host Microbe $2011 ; 9$ : 355-61.

2. Sticher L, Mauch-Mani B, Metraux JP. Systemic acquired resistance. Annu Rev Phytopathol 1997 ; $35: 235-70$.

3. Durrant WE, Dong X. Systemic acquired resistance. Annu Rev Phytopathol 2004 ; 42 : 185-209.

4. Kurtz J, Franz K. Innate defence: evidence for memory in invertebrate immunity. Nature 2003; $425: 37-8$.

5. Little TJ, O'Connor B, Colegrave N, et al. Maternal transfer of strain-specific immunity in an invertebrate. Curr Biol 2003; $13: 489-92$.

6. Sadd BM, Schmid-Hempel P. Insect immunity shows specificity in protection upon secondary pathogen exposure. Curr Biol 2006 ; $16: 1206-10$.

7. Pham LN, Dionne MS, Shirasu-Hiza M, Schneider DS. A specific primed immune response in Drosophila is dependent on phagocytes. PLoS Pathog 2007 ; 3 : e26.

8. Ostuni R, Piccolo V, Barozzi I, et al. Latent enhancers activated by stimulation in differentiated cells. Cell $2013 ; 152: 157-71$.

9. Bistoni F, Vecchiarelli A, Cenci $\varepsilon$, et al. Evidence for macrophage-mediated protection against lethal Candida albicans infection. Infect Immun 1986; $51:$ 668-74.

10. Bistoni F, Verducci G, Perito $S$, et al. Immunomodulation by a low-virulence, agerminative variant of Candida albicans. Further evidence for macrophage activation as one of the effector mechanisms of nonspecific anti-infectious protection. J Med Vet Mycol $1988 ; 26: 285$-99.

11. Vecchiarelli A, Cenci $\varepsilon$, Puliti $M$, et al. Protective immunity induced by low-virulence Candida albicans: cytokine production in the development of the anti-infectious state. Cell Immunol $1989 ; 124: 334-44$.

12. Quintin J, Saeed S, Martens JH, et al. Candida albicans infection affords protection against reinfection via functional reprogramming of monocytes. Cell Host Microbe 2012 ; 12 : 223-32.

13. Kleinnijenhuis J, Quintin J, Preijers F, et al. Bacille Calmette-Guerin induces NOD2-dependent nonspecific protection from reinfection via epigenetic reprogramming of monocytes. Proc Natl Acad Sci USA 2012 ; 109 : 17537-42.

14. Sun JC, Beilke JN, Lanier LL. Adaptive immune features of natural killer cells. Nature 2009 ; 457 : 557-61.

15. Polycarpou A, Holland MJ, Karageorgiou I, et al. Mycobacterium leprae activates Toll-like receptor-4 signaling and expression on macrophages depending on previous bacillus CalmetteGuerin vaccination. Front Cell Infect Microbiol 2016; $6: 72$.

16. Kleinnijenhuis J, Quintin J, Preijers F, et al. Long-lasting effects of BCG vaccination on both heterologous Th1/Th17 responses and innate trained immunity. J Innate Immun $2014 ; 6$ : 152-8.

17. Kumagai $Y$, Akira $S$. Identification and functions of pattern-recognition receptors. J Allergy Clin Immunol $2010 ; 125: 985-92$.

18. Quintin J, Cheng SC, van der Meer JW, Netea MG. Innate immune memory: towards a better understanding of host defense mechanisms. Curr Opin Immunol $2014 ; 29: 1-7$.
19. Margueron R, Reinberg D. Chromatin structure and the inheritance of epigenetic information. Nat Rev Genet $2010 ; 11: 285-96$.

20. O'Neill LA, Hardie DG. Metabolism of inflammation limited by AMPK and pseudo-starvation. Nature $2013 ; 493$ : 346-55.

21. Donohoe DR, Bultman SJ. Metaboloepigenetics: interrelationships between energy metabolism and epigenetic control of gene expression. J Cell Physiol $2012 ; 227: 3169-77$.

22. Liu TF, Yoza BK, દl Gazzar M, et al. NAD+-dependent SIRTl deacetylase participates in epigenetic reprogramming during endotoxin tolerance.J Biol Chem $2011 ; 286$ : 9856-64.

23. Liu TF, Vachharajani VT, Yoza BK, McCall CE. NAD+-dependent sirtuin 1 and 6 proteins coordinate a switch from glucose to fatty acid oxidation during the acute inflammatory response. J Biol Chem 2012 ; 287 : 25758-69.

24. Hoffmann JA. The immune response of Drosophila. Nature $2003 ; 426: 33-8$.

25. Hildemann WH, Johnson IS, Jokiel PL. Immunocompetence in the lowest metazoan phylum: transplantation immunity in sponges. Science 1979 ; $204: 420-2$.

26. Hildemann WH, Raison RL, Cheung G, et al. Immunological specificity and memory in a scleractinian coral. Nature $1977 ; 270: 219-23$.

27. Cooper $\varepsilon L$. Transplantation immunity in annelids. I. Rejection of xenografts exchanged between Lumbricus terrestris and Eisenia foetida. Transplantation $1968 ; 6: 322-7$.

28. Kurtz J. Specific memory within innate immune systems. Trends Immunol $2005 ; 26: 186-92$.

29. Faulhaber LM, Karp RD. A diphasic immune response against bacteria in the American cockroach. Immunology $1992 ; 75$ : 378-81.

30. Roth 0, Kurtz J. Phagocytosis mediates specificity in the immune defence of an invertebrate, the woodlouse Porcellio scaber (Crustacea: Isopoda). Dev Comp Immunol 2009 ; 33 : 1151-5.

31. Rowley AF, Powell A. Invertebrate immune systems specific, quasi-specific, or nonspecific? J Immunol 2007 ; 179 : 7209-14.

32. Morita M. Structure and function of the reticular cell in the planarian Dugesia dorotocephala. Hydrobiologia 1995 ; 305 : 189-96.

33. Moret $Y$, Schmid-Hempel P. Immune defence in bumble-bee offspring. Nature $2001 ; 414: 506$

34. Sadd BM, Kleinlogel Y, Schmid-Hempel R, Schmid-Hempel P. Transgenerational immune priming in a social insect. Biol Lett $2005 ; 1: 386-8$.

35. Baguna J, Salo $\varepsilon$, Auladell C. Regeneration and pattern-formation in planarians. 3. Evidence that neoblasts are totipotent stem-cells and the source of blastema cells. Development $1989 ; 107: 77-86$.

36. Rebecchi L, Altiero T, Guidetti R, et al. Tardigrade resistance to space effects: first results of experiments on the LIFE-TARSE mission on FOTON-M3 (September 2007). Astrobiology 2009; 9 : 581-91.

37. Horikawa DD, Yamaguchi A, Sakashita T, et al. Tolerance of anhydrobiotic eggs of the Tardigrade Ramazzottius varieornatus to extreme environments. Astrobiology $2012 ; 12: 283-9$.

38. Horikawa DD, Cumbers J, Sakakibara I, et al. Analysis of DNA repair and protection in the Tardigrade Ramazzottius varieornatus and Hypsibius dujardini after exposure to UVC radiation. PLoS One $2013 ; 8$ : e64793.

39. Royet J. Les protéines PGRP, un chaînon manquant de l'immunité innée de la drosophile. Med Sci (Paris) $2001 ; 17$ : 1359-62.

40. Cédric Torre $C$, Ghigo $\varepsilon$. La planaire : un ver immortel pour élucider la réponse immunitaire de l'homme. Med Sci (Paris) $2015 ; 31: 20-2$.

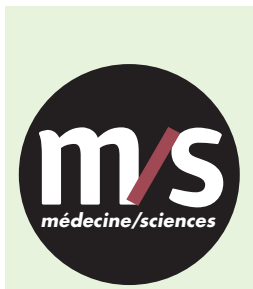

$>$ Grâce à $m / s$, vivez en direct les progrès

Tarifs d'abonnement $\mathrm{m} / \mathrm{s}-2017$ des sciences biologiques et médicales

Abonnez-vous

à médecine/sciences

Bulletin d'abonnement page 1010 dans ce numéro de $\mathrm{m} / \mathrm{s}$
TIRÉS À PART

દ. Ghigo 